\title{
Caso clínico de una paciente diagnosticada de trastorno bipolar desde la visión del proceso enfermero.
}

Case report of a patient diagnosed with bipolar disorder from the perspective of the nursing process.

\author{
Karina R. Campoverde Espinosa ${ }^{a}$, Sergi Balcells Borràs ${ }^{\text {, }}$, Carolina Miguel García ${ }^{\mathrm{c}}$. \\ ${ }^{a, b}$ Enfermeros residentes de la especialidad de Salud Mental. Benito Menni, Complex Assistencial. St Boi \\ de Llobregat, España. ${ }^{c}$ Profesora asociada de la Escuela de Enfermería de la Universidad de Barcelona. \\ Enfermera especialista en Salud Mental.
}

Correspondencia: Karina R. Campoverde Espinosa (hospital@hospitalbenitomenni.org)

Recibido: 03/01/2013; aceptado con modificaciones: 11/05/2013

\begin{abstract}
RESUMEN: La aplicación del método científico en la práctica asistencial llevada a cabo por las enfermeras, se realiza a través del proceso enfermero. Por ese motivo, el propósito de esta nota clínica consiste en destacar, en qué consiste este proceso enfermero, exponiendo el caso clínico de una paciente afectada de Trastorno Mental Grave. Del mismo modo, se destaca la importancia de la adopción de un modelo de cuidados, por lo que nosotros utilizaremos el Modelo de Vida de Nancy Roper, obteniendo de esta manera cuidados enfermeros de calidad.

PALABRAS CLAVE: Diagnóstico de enfermería, metodología enfermera, cuidados de Enfermería; NANDA, NOC, NIC.
\end{abstract}

\begin{abstract}
The application of the scientific method in clinical practice carried out by nurses is done through the Nursing Process. For this reason, the aim of this report is to highlight what NP means, and then, we are going to proceed with a brief explanation of this referred process, exposing, at the same time, the clinical case of a patient affected by severe mental disorder. Similarly, we must remark the importance of adopting a model of care, using the Pattern of Life by Nancy Roper, since this way, we can get to achieve quality nursing care.

KEY WORDS: Nursing Diagnosis, nursing methodology, nursing care; NANDA, NOC, NIC.
\end{abstract}

El caso clínico, expuesto a continuación, es la revisión del proceso enfermero (PE) que se ha seguido desde el Centro de Salud Mental de Adultos (CSMA), Unidad de Agudos y Unidad de Psicogeriatra del centro asistencial Benito Menni de Sant Boi del Llobregat, Barcelona, de una paciente de 65 años, afectada de Trastorno Mental Grave (TMG) y cómo ha sido su evolución desde la visión enfermera.

El PE es un método sistemático, estructurado en etapas ordenadas lógicamente, utilizado para planificar cuidados enfermeros individualizados y deliberados. Este proceso se caracteriza por ser sistemático, dinámico, interactivo pactándolo con el propio paciente, humanístico y centrado en los objetivos (resultados) del paciente.

Los elementos del PE generalmente aceptados son: Valoración, Diagnóstico, Identificación de objetivos, Planificación, Implementación y Evaluación (1).

La valoración enfermera consta de datos subjetivos procedentes de la persona o familiares y de datos objetivos procedentes de pruebas diagnósticas y otras fuentes de datos.

Nuestro marco de valoración enfermero usado para generar diagnósticos enfermeros precisos, son las doce Actividades de la vida diaria (AVD) del denomina- 
do Modelo de Vida de Nancy Roper (2). Estas 12 AVD se miden en un continuum que se mueve desde la dependencia total a la independencia total (Figura1) (3).

Figura 1.

Modelo de Vida de Nancy Roper.

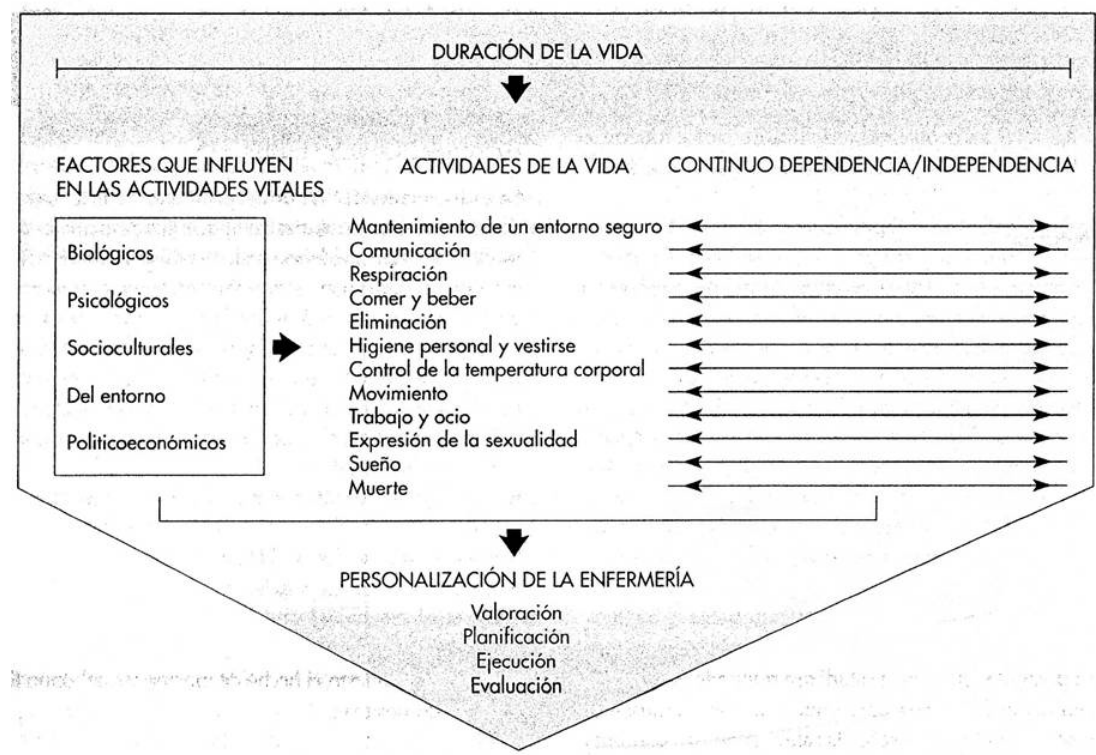

Figura 20-2 Diagrama del modelo de la enfermeria. (De Roper, N., Logan, W.W., Tierney, A.J. [1996]. The Elements of Nursing: A model for nursing based on a model of living [4.a ed., p. 20]. Edimburgo: Churchill Livingstone. Utilizado con permiso de Churchill Livingstone.)

El diagnóstico enfermero, asociados a la valoración enfermera y basado en el juicio clínico, se formulan mediante la Taxonomía de la NANDA II, 2009-2011 (4). Esta taxonomía consta de 13 dominios representando un área de funcionamiento y/o comportamiento, 47 clases y 206 diagnósticos de enfermería.

Una vez realizados los diagnósticos enfermeros con los objetivos identificados, se procede a la planificación de los cuidados o tratamientos enfermeros, es decir las actividades enfermeras con sus respectivas intervenciones, para reducir, eliminar o prevenir los problemas detectados en la etapa anterior.

La Clasificación de Intervenciones Enfermeras (NIC) (5), constituido por 542 intervenciones y 12.000 actividades, son cualquier tratamiento, basado en el juicio clínico y el conocimiento, que proporciona a la enfermera una forma de atención individualizada, incluyendo actividades tanto generales como especializadas.

La Clasificación de Resultados Enfermeros (NOC) (6) son una clasificación global y estandarizada de los resultados del paciente, teniendo en cuenta que lo que 
NOTAS CLÍNICAS

se pretende con los objetivos enfermeros es establecer: qué se quiere conseguir, cómo, cuándo y cuánto. En total encontramos 385 resultados.

Una vez empleada las taxonomías NANDA-II, NOC y NIC, procedemos a la implementación de los cuidados seleccionados dentro del plan de cuidados.

Finalmente, se procede a la evaluación para la comparación de los resultados obtenidos con los objetivos propuestos. En este paso identificaremos las variables que afectan el logro de los objetivos y decidiremos si hay que mantener, modificar o dar por terminado el plan.

Una vez explicado el PE, se procede a la explicación del caso clínico de la paciente, diagnosticada según criterios del DSMIV-TR (7) de Trastorno Bipolar tipo I, episodio más reciente mixto, grave con síntomas psicóticos.

Para la valoración se utiliza el Modelo de Vida de Nancy Roper, tal como se había explicado anteriormente, destacando como antecedentes:

\section{Somático:}

No padece alergias medicamentosas conocidas.

Cirrosis hepática con varices esofágicas. Pancreatitis crónica.

Osteoporosis

Hipotiroidismo subclínico por Lítio (tratamiento sustitutivo con hormona tiroidea).

\section{Tóxicos:}

Dependencia alcohólica desde los 20 años. Abstinente desde hace años, con consumos puntuales durante las descompensaciones. Antecedentes de abuso de anfetaminas, hipnosedantes, estando actualmente abstinente.

\section{Sociales:}

Separada y con dos hijos. Convivía con su pareja estable desde hace años, hasta el fallecimiento reciente de éste. Padres con enolismo, ya fallecidos y hermano con dependencia alcohólica en remisión.

\section{Psiquiátricos:}

- Inicio de trastorno a los 28 años orientándose como Trastorno Límite de la Personalidad (TLP).

- En 1976 ingresa en Hospital Psiquiátrico para desintoxicación de alcohol, con posterior seguimiento psicoterapéutico de grupo.

- Entre los años 1978 y 1986 requiere de varios ingresos en la Unidad de Agudos de Psiquiatría por:

Alcoholismo, personalidad patológica borderline, conductas impulsivas e impredecibles y estado de ánimo inestable. 
- Desde 1992 hasta 2005 ingresa en Unidad de Larga Estancia de Hospital Monográfico mostrando importantes alteraciones de conducta secundarias a rasgos de personalidad vs sintomatología afectiva e ingesta enólica siendo diagnosticada finalmente de Trastorno Bipolar I.

- En el 2005, se da el alta de la Unidad de Larga Estancia, ya que contaba con una estabilidad relativa, teniéndose en cuenta el apoyo de contención familiar (su pareja que conoce en uno de los permisos del ingreso). Fue vinculada al CMSA con Psiquiatría y Enfermería de Salud Mental, mostrando buena adherencia.

- Desde el 2006 hasta el 2010 requiere de varios ingresos en la Unidad de Agudos de Psiquiatría, por:

Sintomatología ansiosa, sobreingesta medicamentosa (SIM), descompensaciones maniformes y alteraciones conductuales e intoxicación etílica.

- En el 2011 realiza una SIM, que no requiere ingreso hospitalario, por lo que desde el CSMA es derivada a la Unidad de Rehabilitación.

- En febrero de 2012 ingresa en la Unidad de Agudos de Psiquiatría, por recaída depresiva debido a la muerte reciente de su pareja. Durante este ingreso se realizan sesiones de terapia electroconvulsiva.

En marzo de este mismo año hasta la actualidad, es derivada a la Unidad de Psicogeriatría para estabilización psicopatológica y elaboración de un plan de recaídas.

Dentro de las 12 AVD del Modelo de Nancy Roper (8), se consideran que deben ser tratadas de manera prioritaria el mantenimiento del entorno seguro y trabajo/diversión, tal como se recoge en la tabla 1 y 2.

A modo de conclusión, se observa por un lado que con el PE se desarrolla una base de conocimientos propia, organizando el proceso de actuación y aunando la manera de trabajar de los profesionales enfermeros, con el objetivo tanto de una autonomía como reconocimiento social enfermero, aportando también una visión en todas las esferas (bio-psico-sociales) pudiendo ser beneficiosa para otros profesionales en el desarrollo de la práctica multidisciplinar (médicos, psicólogos, trabajadores sociales, etc.). Por otra parte, también beneficia a la paciente porque la hacemos partícipe de los cuidados llevados a cabo, mejora su autonomía en el manejo de las AVDs, su autoestima y motivación para la adquisición de mejoras en sus hábitos de salud y su patología en global. 
NOTAS CLÍNICAS

Tabla 1.

Diagnósticos - Resultados - Intervenciones.

\begin{tabular}{|c|c|c|}
\hline $\begin{array}{l}\text { Diagnósticos enfermero. } \\
\text { NANDA. }\end{array}$ & Resultados. NOC. & Intervenciones. NIC. \\
\hline
\end{tabular}

Tabla 2.

Diagnósticos - Resultados - Intervenciones.

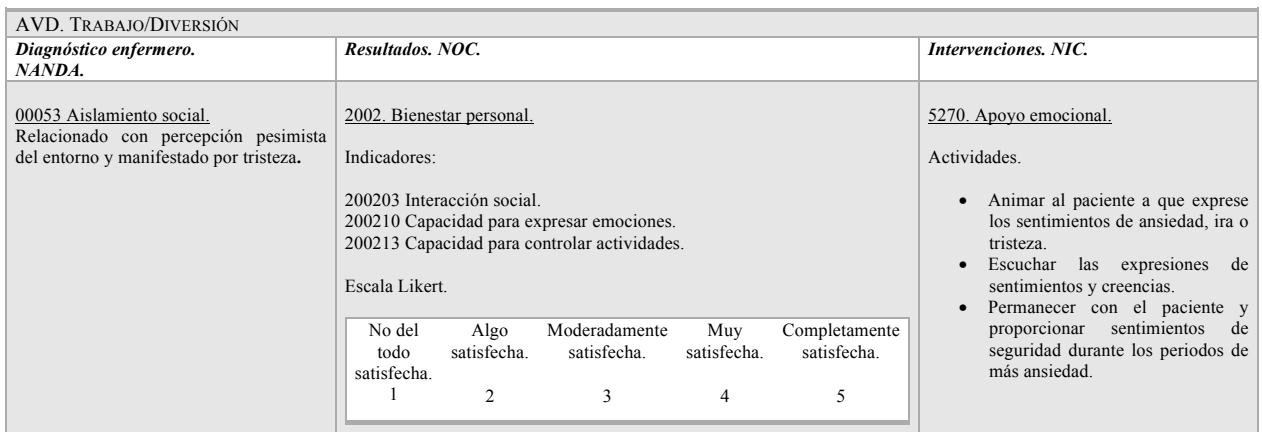




\section{BIBLIOGRAFIA:}

(1) Silver Spring, MD: ANA Nursing: Scope and standards of practice 2nd ed. American Nurses`Association 2010.

(2) Roper, N. Winifred, W. L. Tierney, A.J. Modelo de enfermería: basado en el modelo de vida. $2^{\mathrm{a}}$ ed. Mexico D.F: Interamericana McGraw-Hill, 1993.

(3) Roper, N. Winifred, W. L. Tierney, A.J. The Elements of Nursing: A model for nursing base on a model of living. $4^{\circ} \mathrm{ed}$. Edinburgh: Churchill Livingstone, 1996.

(4) Heather Herdman, T; Heath, C; Lunney, M; Scroggins, L; Vassallo, B. Libro de la NANDA internacional 2009-2011. Barcelona: Elsevier Mosbi; 2010.

(5) M. Bulechek, G; K. Butcher, H; McCloskey Dochterman, J. Clasificación de intervenciones de enfermería (NIC). $5^{a}$ ed. Barcelona: Elsevier Mosbi; 2009.

(6) Moorhead, S; Johnson, M; L. Maas, M; Swanson, E. Clasificación de resultados de enfermería (NOC). $4^{\mathrm{a}}$ ed. Barcelona: Elsevier Mosbi; 2009.

(7) López-Ibor Aliño, J. J.; Valdés Miyar, M. (dir.) DSM-IV-TR. Manual diagnóstico y estadístico de los trastornos mentales. Texto revisado. $1^{\mathrm{a}}$ ed. Barcelona: Masson; 2002.

(8) Benavent Garces, MA; Ferrer Ferrandis, E; Francisco del Rey, C. Corrientes del pensamiento enfermero II: modelos y teorias (1970 hasta la actualidad). En: Benavent Garces, MA; Ferrer Ferrandis, E; Francisco del Rey, C. Fundamentos de Enfermeria. Coleccion Enfermeria S21. $2^{\mathrm{a}}$ ed. Madrid: Difusion Avances de Enfermeria (DAE); 2009. p. 204-223. 